\title{
Control of brain state transitions with light
}

Almudena Barbero-Castillo ${ }^{1,+}$, Fabio Riefolo ${ }^{2,3,+}$, Carlo Matera ${ }^{2,3}$, Sara Caldas-Martínez ${ }^{1}$, Pedro Mateos-

Aparicio $^{1}$, Julia F. Weinert ${ }^{1}$, Enrique Claro ${ }^{4}$, Maria Victoria Sánchez-Vives ${ }^{1,5, *}$, and Pau Gorostiza ${ }^{2,3,5,{ }^{*}}$

1. Institut d'Investigacions Biomèdiques August Pi i Sunyer (IDIBAPS), Barcelona, Spain

2. Institute for Bioengineering of Catalonia (IBEC), Barcelona Institute for Science and Technology (BIST),

Barcelona, Spain.

3. Network Biomedical Research Center in Bioengineering, Biomaterials, and Nanomedicine (CIBER-BBN),

Madrid, Spain.

4. Institut de Neurociències and Departament de Bioquímica i Biologia Molecular, Unitat de Bioquímica de Medicina, Universitat Autònoma de Barcelona (UAB), Barcelona, Spain.

5. Catalan Institution for Research and Advanced Studies (ICREA), Barcelona, Spain.

† Equal contribution.

* Corresponding authors. E-mail: msanche3@clinic.cat (M.V.S.V.), pau@icrea.cat (P.G.) 


\section{ABSTRACT}

Behavior is driven by specific neuronal activity and can be directly associated with characteristic brain states.

The oscillatory activity of neurons contains information about the mental state of an individual, and the transition between physiological brain states is largely controlled by neuromodulators. Manipulating neural activity, brain rhythms or synchronization is of significant therapeutic interest in several neurological disorders and can be achieved by different means such as transcranial current and magnetic stimulation techniques, and by light through optogenetics, although the clinical translation of the latter is hampered by the need of gene therapy. Here, we directly modulate brain rhythms with light using a novel photoswitchable muscarinic agonist. Synchronous slow wave activity is transformed into a higher frequency pattern in the cerebral cortex both in slices in vitro and in anesthetized mice. These results open the way to the study of the neuromodulation and control of spatiotemporal patterns of activity and pharmacology of brain states, their transitions, and their links to cognition and behavior, in different organisms without requiring any genetic manipulation.

\section{INTRODUCTION}

All perceptions, memories, and behaviors are rooted in the communication between the billions of neurons that constitute the brain. ${ }^{1-3}$ Individual neurons transmit information using chemical and electrical signals, and are organized in groups or circuits involved in different functions. ${ }^{3-5}$ The electrochemical interactions of these neuronal ensembles produce synchronized electrical activity in the brain, that constitute brain rhythms or oscillations due to their periodic properties, and brain waves when those oscillations show spatial propagation. ${ }^{6-8}$ Brain rhythms can be recorded by different means, involving largely electrophysiological (e.g. electroencephalography (EEG), local field potential (LFP), etc.) or imaging methods. Brain rhythms, their frequency, synchronization or functional connectivity associated to them, are associated 
with internal brain states and result in specific behaviors. ${ }^{1}$ Neuronal and network activity undergoes significant changes during brain and behavioral state transitions, which have been linked to substantial changes in EEG pattern activity. ${ }^{9}$ For example, large and synchronous brain waves are mostly associated with deep sleep, whereas in wakefulness there is a shift towards more desynchronized and short-amplitude wave patterns. ${ }^{7,10,11}$ These changes in brain and behavioral states, and the concomitant alterations in EEG activity, can be driven by the action of neuromodulators like acetylcholine (ACh). ${ }^{9,12}$ However, it is not fully known how the different cells expressing ACh receptors contribute to the alteration of the global cortical state. Cholinergic receptors include ionotropic nicotinic ion channels and muscarinic metabotropic $\mathrm{G}$ proteincoupled receptors. Together, they modulate cortical activity on a fine spatial scale and are involved in crucial neocortical functions such as attention, ${ }^{13,14}$ learning, ${ }^{15-17}$ memory, ${ }^{18}$ and sensory and motor functions. ${ }^{19,20}$ In the neocortex, ACh is released mostly at cholinergic afferents from neurons distributed within the basal forebrain (BF) nuclei. Electrical stimulation of the nucleus basalis can evoke the release of $\mathrm{ACh}$ in the neocortex but in an unselective manner, as ascending projections from BF nuclei not only comprise cholinergic axons, but also GABAergic and glutamatergic axons. ${ }^{21}$ Such a lack of selectivity complicates the study of cholinergic signaling in the neocortex and its effects on controlling brain states.

Selective stimulation of cholinergic projections in the neocortex from BF nuclei has been demonstrated with optogenetics, which enables the disruption of neocortical synchronous activity during certain sleep states. Optogenetic stimulation of BF cholinergic neurons also revealed their influence in awake cortical dynamics, coding properties of V1 neurons, and the importance of cholinergic neuromodulation for visual discrimination tasks, showing that stimulation of $\mathrm{BF}$ cholinergic cells activates cortical transitions faster than previously presumed. ${ }^{10}$ However, the cell type specificity of optogenetics is limited by the availability of suitable promoters. In addition, it is based on the overexpression of microbial proteins using genetic manipulation, which can distort synaptic physiology. ${ }^{22-24}$ This raises safety and regulatory concerns regarding therapeutic applications. 
The control of neuronal signaling with photopharmacology is based on synthetic ligands that target endogenous proteins, and thus its physiological relevance spans from circuit to sub-cellular levels. Since neuronal receptors are highly conserved, photoswitchable ligands can generally be used in multiple species, and their safety and regulation can be established in the same manner as other drugs. The cholinergic system is key to the modulation of a variety of CNS functions, ${ }^{25}$ and thus the use of selective and photoswitchable cholinergic drugs to spatiotemporally control cortical activity could have relevant scientific and clinical implications. Herein, we report that the cholinergic-dependent brain state transitions in the neocortex can be directly controlled using Phthalimide-Azo-Iper (PAI) ${ }^{26}$ a photoswitchable agonist that targets M2 muscarinic acetylcholine receptors (mAChRs) without requiring electric or genetic manipulation. In particular, PAI enables the modulation of spontaneous emerging slow oscillations (SO) in neuronal circuits. PAl cis-to-trans photoisomerization decreases the Down- and Up-state durations and increases oscillatory frequency in cortical slices. In addition, PAl allows the reversible manipulation of the cortical oscillation frequency in anesthetized mice using light. Thus, photopharmacology allows the selective control of slow oscillations in vitro and in vivo, opening the way to the analysis of their spatiotemporal dynamics and their effects on brain and behavioral state transitions.

\section{RESULTS}

\section{Non-specific activation of mAChRs evokes neuronal hyperexcitability in cortical slices}

Changes in cortical rhythms underlie behavioral state transitions, and endogenous ACh actions play a central role in such variations. ${ }^{27-33}$ However, a complete and unifying view has not yet emerged ${ }^{11}$ regarding the cholinergic impact on neuronal and synaptic physiology, and thus on neocortical network dynamics. It is known that ACh contributes to the shift of the neocortical network state from synchronous to asynchronous activity -associated to awake state- in a dose-dependent manner, but the activity of ACh in the vast majority of neocortical neurons and synapses is still poorly characterized. Neocortical activation of mAChRs in vitro facilitates synaptic transmission, ${ }^{34}$ recurrent excitation, ${ }^{35}$ and reversibly increases the power of fast- 
frequency oscillations. ${ }^{36-39}$ On these basis, we first studied in vitro the effect of Iperoxo, a potent muscarinic agonist. ${ }^{40,41}$ The goal was to evaluate the potential of mAChRs to selectively modulate the dynamics of isolated neocortical slices, while avoiding the simultaneous activation of nicotinic cholinergic receptors (nAChRs), and to validate Iperoxo photoswitches and useful photopharmacological tools to control neuronal activity. Isolated cortical slices spontaneously generate cortical SO, a hallmark of activity during deep sleep or anesthesia. ${ }^{42}$ We recorded the spontaneous oscillatory activity (control) and then the activity under different concentrations $(1,10,100 \mathrm{nM})$ of Iperoxo. The spontaneous slow oscillatory activity alternates between periods of activity or high neuronal firing (Up states) and periods of near silence (Down states). The activation of mAChRs by Iperoxo resulted in a global change in the network's dynamics to a hyperexcitable state (Fig. 1). At $100 \mathrm{nM}$ Iperoxo, the oscillatory frequency increased (from $0.89 \pm 0.12 \mathrm{~Hz}$ in the control, to $1.34 \pm 0.18 \mathrm{~Hz}$ with $100 \mathrm{nM}$ Iperoxo), and the relative firing rate ${ }^{43}$ of the Up-states decreased (from $1.13 \pm$ 0.23 a.u. to $0.10 \pm 0.02$ with $100 \mathrm{nM}$ Iperoxo) (Fig. 1B). Furthermore, at concentrations equal or higher than $100 \mathrm{nM}$ Iperoxo, the oscillatory activity evolved to periods of seizure-like discharges (Fig.1C), ${ }^{44,45}$ characterized by low ( $<1 \mathrm{~Hz})$, delta (1-4 Hz) and alpha-beta (8-16 Hz) frequencies (Fig. 1D). 
A

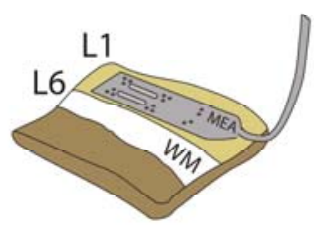

B

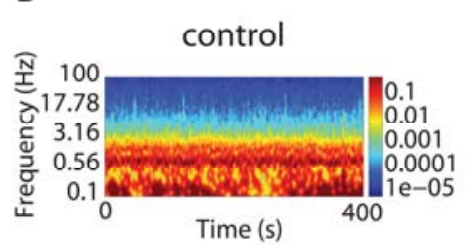

IPX $100 \mathrm{nM}$

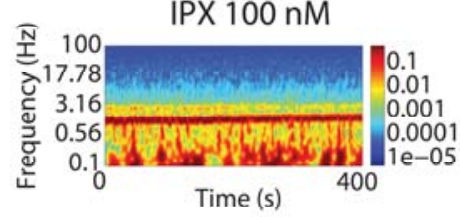

Epilepsy (IPX 100 nM)

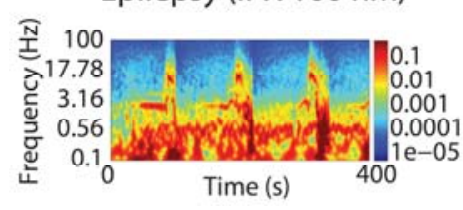

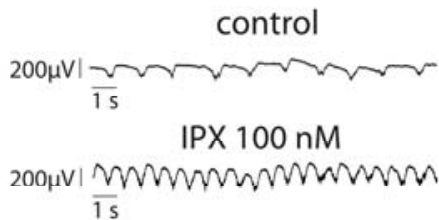

C

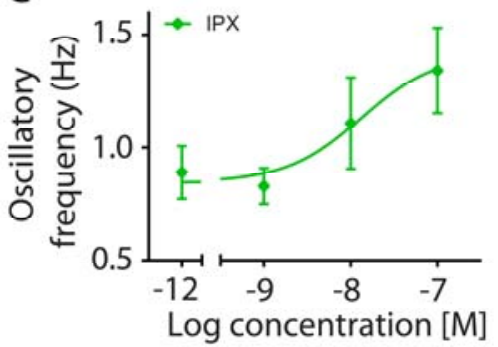

D

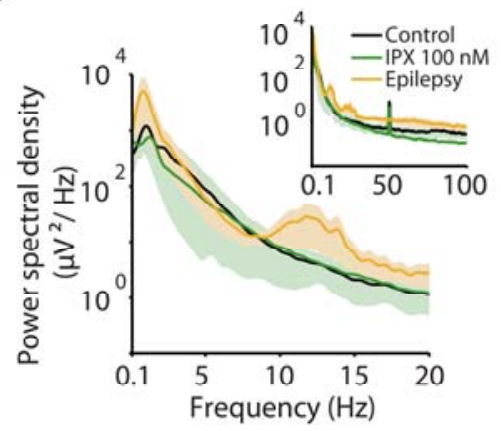

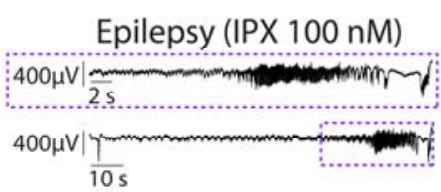
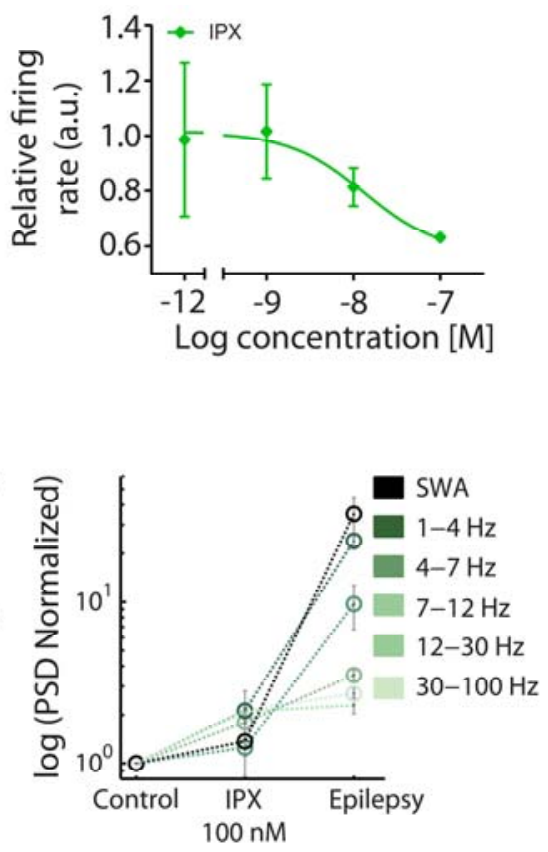

Figure 1: Non-specific activation of mAChRs evokes neuronal hyperexcitability in cortical slices. (A) On the left, the experimental setup: MEA, multielectrode array; WM, white matter; L1-L6, layer 1-6. On the right, representative Local Field Potential (LFP) traces showing the increasing of the OF corresponding to the Spectrogram of panel B. (B) Spectrogram under color from the same time recording of LFP traces on panel A: control, $100 \mathrm{nM}$ Iperoxo and periods seizure-like discharges. (C) OF (Hz) and relative firing rate (a.u.). (D) Power spectral density (PSD) values showing low, delta and theta frequency component. Normalized PSD values at different frequency bands. 


\section{Effect of PAI isomers on slow and fast oscillations in vitro}

The hyperexcitable network state induced with Iperoxo reflects the impact of mAChRs activation on cortical networks and brain states. ${ }^{11}$ In order to remotely control these states, we aimed at the observed muscarinic neuromodulation using PAl, a novel photoswitchable Iperoxo derivative that allows the reversible activation of $\mathrm{M} 2 \mathrm{mAChRs}$ with light. ${ }^{26} \mathrm{M} 2$ receptors play a relevant role in several CNS disorders, ${ }^{25}$ and regulating their activity and subsequent effects on cortical neuronal networks may provide new therapeutic opportunities for these diseases involving the cholinergic system.

Taking advantage of its profile as a M2 light-regulated agonist, we studied its effect on the modulation of neuronal oscillations in cortical slices. Thus, we applied PAI to spontaneously active neocortical brain slices and recorded their oscillatory activity before and after activating the drug by means of illumination. We first generated the dose-response curves of the two drug forms separately, trans- (dark-adapted state) and cisPAI (after UV irradiation), in order to discern the differences in cortical activity between the PAl-isoforms, and to identify the most convenient concentration range to manipulate brain waves with light. The baseline activity (characterized by slow oscillations) was recorded as a control, prior to bath-application of solutions with increasing PAl concentrations $(10 \mathrm{nM}, 100 \mathrm{nM}, 300 \mathrm{nM}$, and $1 \mu \mathrm{M}, n=6$ for each PAl form, trans and cis) (Fig. 2A). Comparing with the control situation, the trans-PAl activity showed alterations in the Up- and Down-state sequence already at $100 \mathrm{nM}$ (Fig.2), leading to an increment in the oscillatory frequency (from $0.58 \pm 0.06 \mathrm{~Hz}$ during control to $1.87 \pm 0.12 \mathrm{~Hz}$ applying $1 \mu \mathrm{M}$ trans-PAl) (Fig. 2B). Moreover, increasing concentrations of trans-PAI decreased the relative firing rate during the Up states (from $0.98 \pm 0.11$ a.u. during control to $0.37 \pm 0.03$ a.u. with $1 \mu \mathrm{M}$ trans-PAl) (Fig. 2C, D). In comparison, cis-PAl displayed significantly weaker effects, in agreement with the reported PAl properties. ${ }^{26}$ At $100 \mathrm{nM}$ and $300 \mathrm{nM}$, cis-PAI did not alter the spontaneous activity observed in control experiments (control oscillatory frequency: $0.48 \pm$ $0.037 \mathrm{~Hz} ; 100 \mathrm{nM}$ cis-PAI OF: $0.52 \pm 0.07 \mathrm{~Hz} ; 300 \mathrm{nM}$ cis-PAl OF: $0.87 \pm 0.2 \mathrm{~Hz}$ ), in contrast to the strong alterations in oscillatory activity obtained with $100 \mathrm{nM}$ and $300 \mathrm{nM}$ trans-PAI (Fig. 2). Only at concentrations as high as $1 \mu \mathrm{M}$, cis-PAl altered the Up- and Down-state sequence in comparison to the control, increasing the oscillatory frequency $(1.32 \pm 0.27 \mathrm{~Hz})$ and decreasing the relative firing rate of the Up-states (from $0.86 \pm$ 
0.04 a.u. during control to $0.74 \pm 0.27$ a.u. with $1 \mu \mathrm{M}$ cis-PAI) (Fig. 2C, D). In summary, the most compelling differences between trans- and cis-PAl emerged between $100 \mathrm{nM}$ and $300 \mathrm{nM}$ and were observed in the oscillatory frequency and in the Up-states' relative firing rate (a.u.) (Fig. 2). We focused on these concentration range therefore in order to photomodulate cortical slow oscillations using PAI.

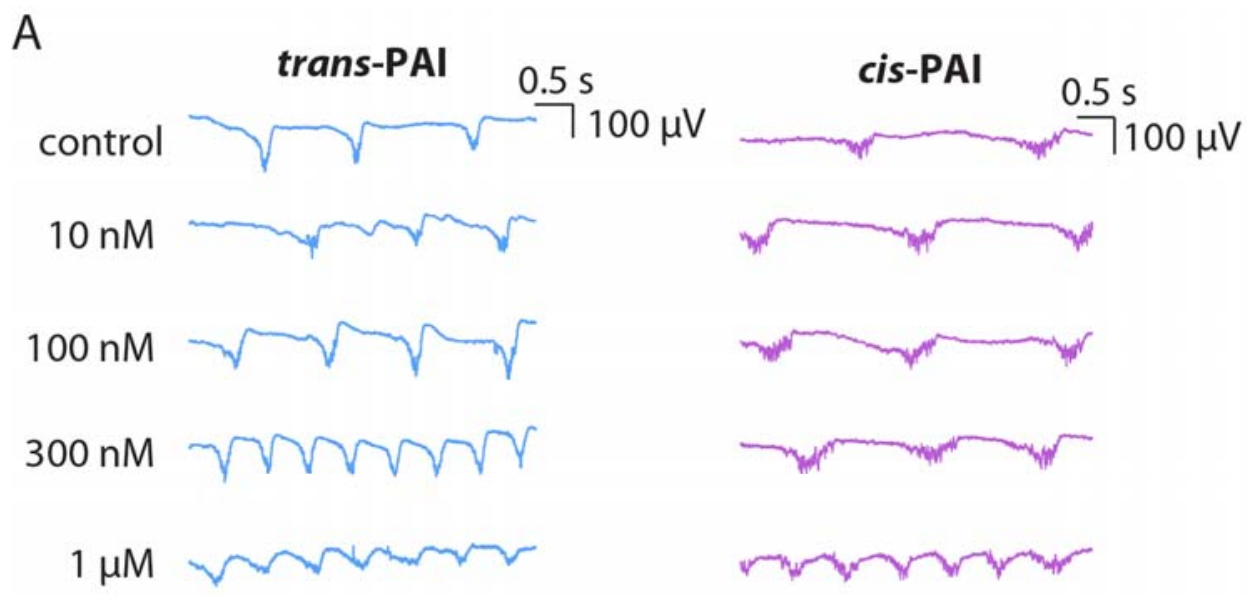

B

trans-PAI
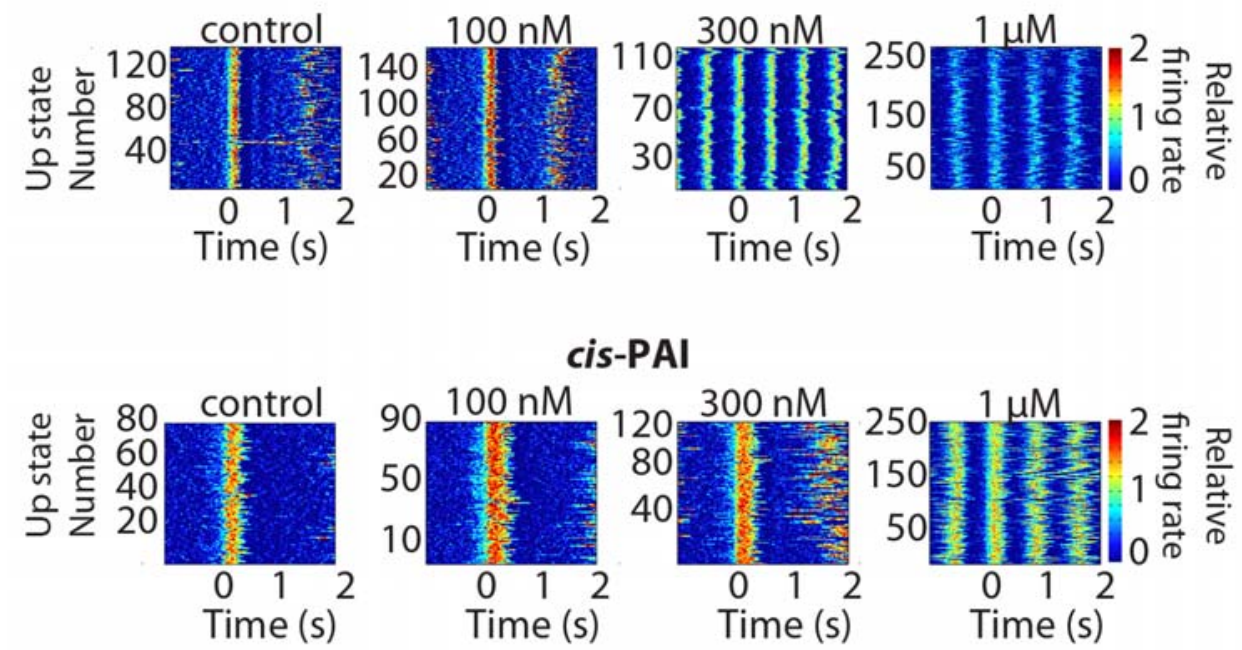

cis-PAI
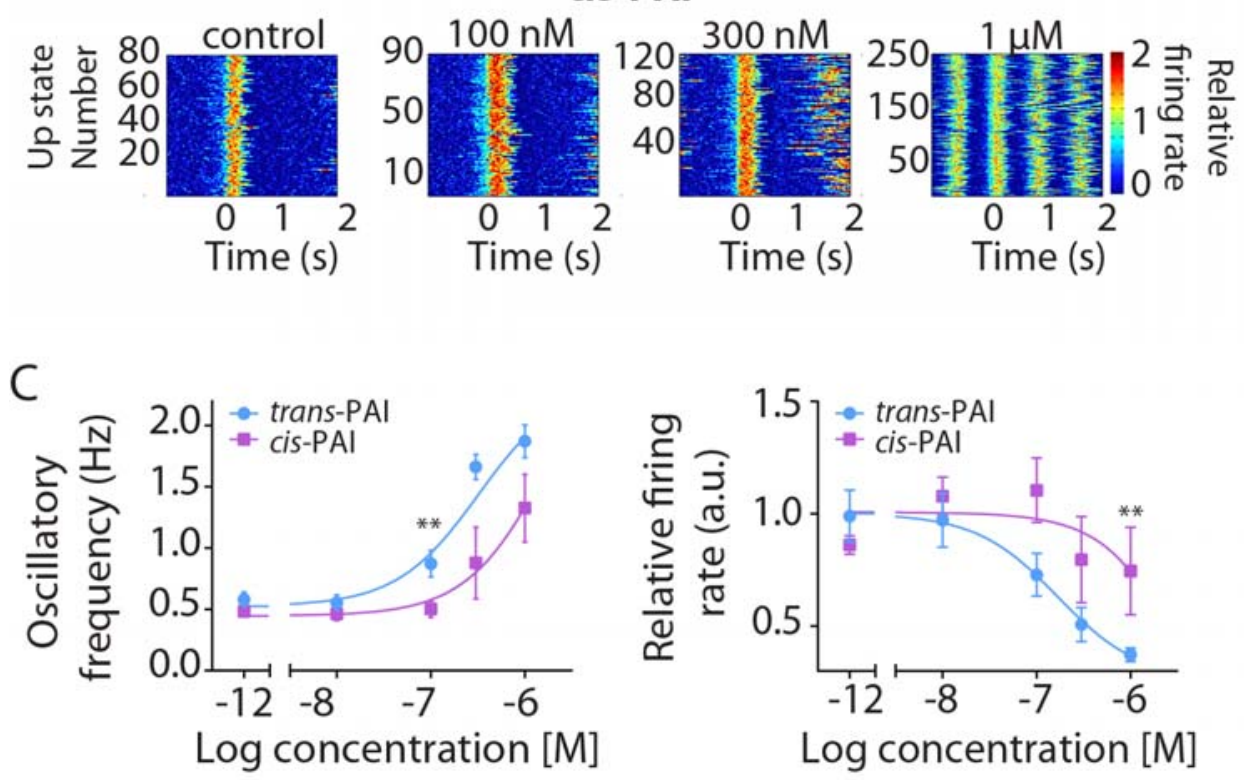
Figure 2: Effect of mAChRs activation by trans-PAI and cis-PAI on SO. (A) Raw LFP example recordings showing the different ability of trans- and cis-PAl to increasing the OF. (B) Raster plots showing the relative firing rate (color coded) under control conditions and different trans- and cis-PAl concentrations. (C) OF ( $\mathrm{Hz})$ and Relative firing rate (a.u.) of the two different PAl isomers, trans- (blue, $\mathrm{n}=6$ ) and cis-PAl (pink, $n=6$ ) at different concentrations. $\left({ }^{* *} p<0.01\right)$.

\section{PAl effectively modulates cortical slow oscillations in vitro}

Once the different oscillatory activity evoked by cis- and trans-PAI was quantified in vitro (Fig.2), we moved on to the control of the rhythmic activity with light in the same cortical slices (Fig.3). We took advantage of the thermal stability of both PAI forms to apply initially the inactive one (cis-PAI) at $200 \mathrm{nM}$ in cortical slices ( $n=15)$, in the absence of white light to avoid photoconversion to trans-PAl during the recordings ${ }^{26}$ As shown in Fig.2, $200 \mathrm{nM}$ cis-PAl evoked an increase in the oscillatory frequency (from $0.53 \pm 0.04 \mathrm{~Hz}$ in control conditions to $1.04 \pm 0.14 \mathrm{~Hz}$ with cis-PAl, $p$-value $=0.0016$ ), and no significant effects in the relative firing rate of the Up-states (from $0.98 \pm 0.09$ a.u. in the control to $0.86 \pm 0.10$ a.u. with cis-PAl, $p$-value $=0.2868$ ) (Fig. 3). Subsequent illumination of the slices with white light produced a robust increase in the oscillatory frequency (from $0.53 \pm 0.04 \mathrm{~Hz}$ in the control to $1.68 \pm 0.13$ upon illumination, $p$-value $=2.93 * 10^{-4}$; from $1.04 \pm 0.14 \mathrm{~Hz}$ with cis-PAl to $1.68 \pm 0.13$ upon illumination, $p$-value $=3.51 * 10^{-4}$ ), a significant decrease in relative firing rate of the Up-states (from control values of $0.98 \pm 0.09$ a.u. to $0.52 \pm 0.06$ a.u. upon illumination, $p$-value $=2.93 * 10^{-4} ;$ from $0.86 \pm 0.10$ a.u. with cis-PAl to $0.52 \pm 0.06$ a.u. upon illumination, $p$ value $=2.93 * 10^{-4}$ ), and a noticiable change in the activity regime of the network (Fig. $3 \mathrm{~A}$ and $\mathrm{B}$ ). The changes in the power spectrum in the population were incremental, as illustrated in the normalized power spectrum (Fig. 3). These changes are similar to the effects of adding Iperoxo to the bath (Fig. 1), and in agreement with PAI photoconversion to the active form (trans). The modification in cortical activity was not reversible with $365 \mathrm{~nm}$ light (to isomerize PAI to the cis form in situ), due either to the irreversibility of muscarinic stimulation in the absence of BF afferents and other circuit components in brain slices, or to neuronal potential changes produced by UV light. 


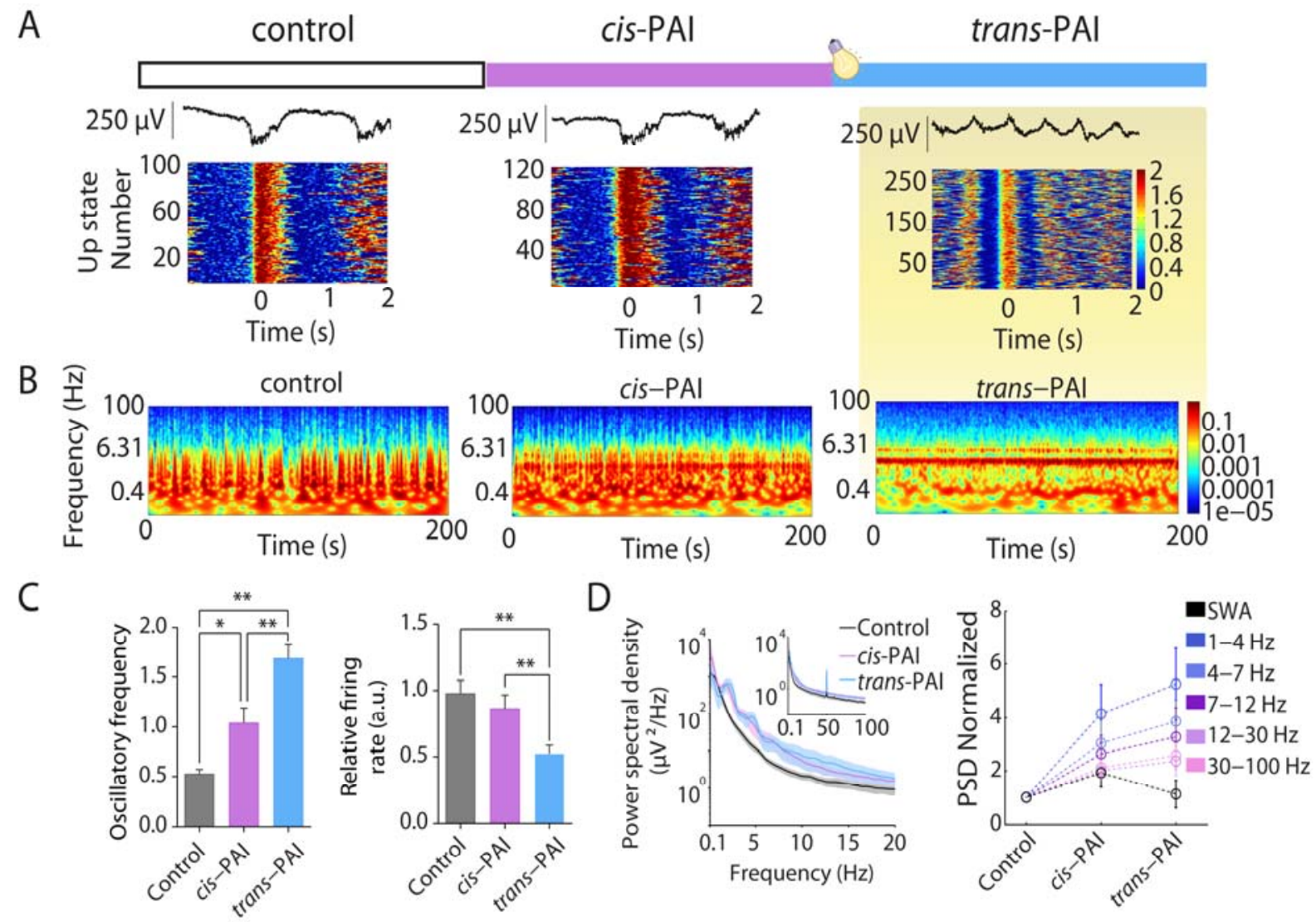

Figure 3: Modulation of brain waves in vitro using PAI, a light-regulated ligand. (A) Representative Local Field

Potential traces (top) and raster plots of relative firing rate under control conditions, $200 \mathrm{nM}$ of cis-PAl and $200 \mathrm{nM}$ trans-PAl after light activation $(n=15)$ (bottom). (B) Representative spectrogram under control condition, $200 \mathrm{nM}$ of cis-PAl and $200 \mathrm{nM}$ trans-PAl after light activation. (C) OF (Hz) and relative firing rate of the Up-states (a.u.) at $200 \mathrm{nM}$ PAl after pre-irradiation at $365 \mathrm{~nm}$ (cis-PAl), and photoswitching with white light (trans-PAl). (D) Averaged Power Spectral Density (PSD) under control conditions, $200 \mathrm{nM}$ of cis-PAl and $200 \mathrm{nM}$ trans-PAl after white light activation (color code). ${ }^{*} p<0.005$ and ${ }^{* *} p<0.001$.

\section{PAl can modulate brain wave activity with light in vivo}

Having established the unique ability of PAI to alter cortical oscillatory activity with light in slices, we then aimed to photocontrol brain state transitions in vivo. Cortical brain waves were recorded in C57BL6/JR mice 
$(n=4)$ with an electrode inserted through a craniotomy across which we carried out the drug application and brain illumination (see Methods). Initially, we induced deep anesthesia in the animals, a state that is known to reproduce the slow wave sleep state, ${ }^{28,29,46}$ and which is characterized by the generation of cortical slow oscillations similar to the slow frequency waves observed in our experiments in slices under control conditions (Fig.1-3). ${ }^{6}$ Such slow oscillations activity in anesthetized mice was recorded for $500 \mathrm{~s}$ under white light illumination of the brain, and the characteristic parameters obtained (oscillatory frequency $0.64 \pm 0.06$ $\mathrm{Hz}$, relative firing rate $0.83 \pm 0.23$ a.u.) were taken as the control condition in vivo. As the dose-response curves of cis- and trans-PAl could be different from the in vitro conditions (Fig. 2), we tested two different concentrations, $200 \mathrm{nM}$ and $1 \mu \mathrm{M} .100 \mu \mathrm{L}$ of $200 \mathrm{nM}$ cis-PAl solution were initially applied to the brain surface, and the activity was recorded for another $500 \mathrm{~s}$ in the absence of white light, to avoid cis to trans photoisomerization of PAI. The oscillatory frequency and the frequencies power of the cortical oscillatory activity was not significantly altered by cis-PAI $(0.61 \pm 0.06 \mathrm{~Hz})$ and caused a minor increase in the relative firing rate (1.02 \pm 0.31 a.u.). Subsequently, we illuminated the brain using white light in the proximity of the recording electrode, in order to isomerize PAI to its active form (trans) and recorded the activity for another $500 \mathrm{~s}$. An increase in the oscillatory frequency to $0.76 \pm 0.1 \mathrm{~Hz}$ and marked power in the $1 \mathrm{~Hz}$ band was observed under white light illumination, without changes in the relative firing rate $(1.04 \pm 0.27$ a.u. $)$. Applying a higher concentration of cis-PAI $(1 \mu \mathrm{M})$ slightly reduced the OF to $0.61 \pm 0.1$, decreased power at $1 \mathrm{~Hz}$ band without significantly affecting the relative firing rate $(0.99 \pm 0.27$ a.u. $)$. However, following illumination, the oscillatory frequency increased to $0.83 \pm 0.06 \mathrm{~Hz}$, appeared again $1 \mathrm{~Hz}$ frequency band and the relative firing rate decreased to $0.86 \pm 0.19$ a.u. (Fig. 4), following the tendency previously observed in vitro. By using two concentrations of the drug, we observed that the effect on the oscillatory frequency was already reached with $200 \mu \mathrm{M}$, while the tendency of the firing rate towards a decrease was more evident under $1 \mu \mathrm{M}$. This strategy allowed us to repeat twice the photocontrol of cortical activity by subsequent application of $1 \mu \mathrm{M}$ cis-PAl and photoconversion to trans-PAl. The extent to which the diffusion of the drug reaches in adequate concentrations the deep layers of the cortex and to which the light reaches such deep 
layers needs further studies, given that the "engine" of slow oscillations lies in deep, layer 5-layer 6 layers. $^{42,47-50}$

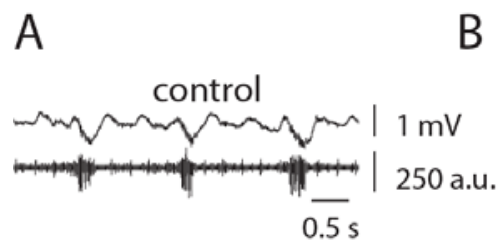

200 nM cis-PAl

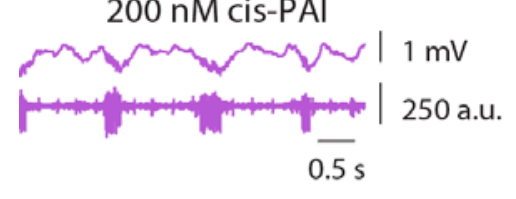

$200 \mathrm{nM}$ trans-PAI

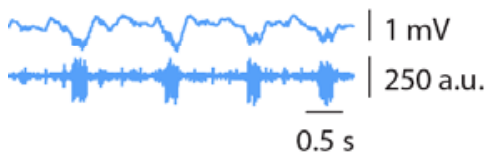

$1 \mu \mathrm{M}$ cis-PAl

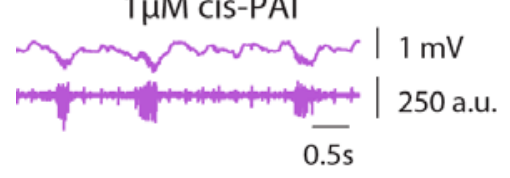

$1 \mu \mathrm{M}$ trans-PAI

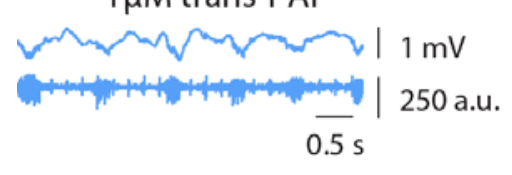

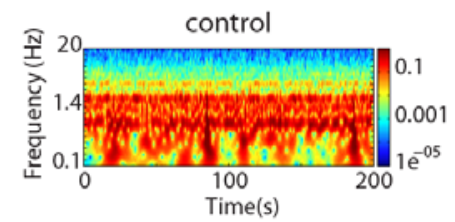
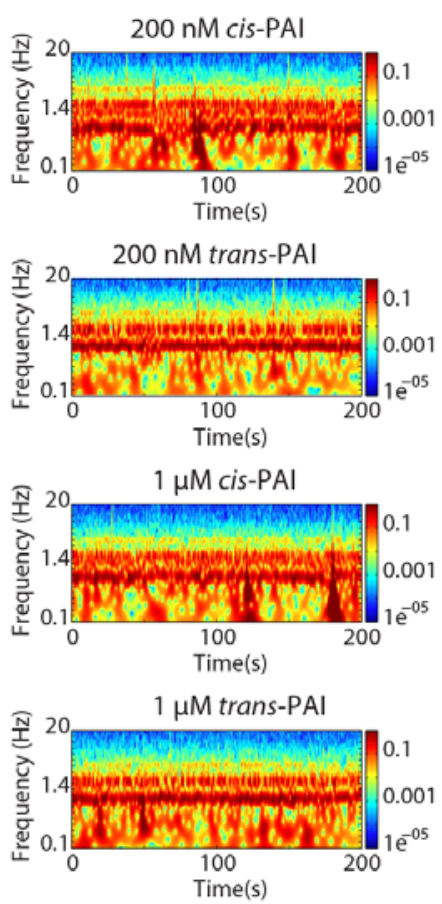

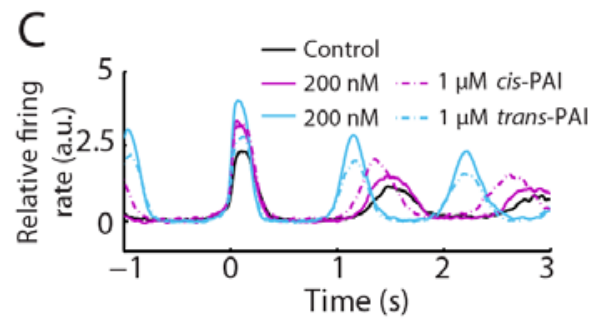

$\mathrm{D}$

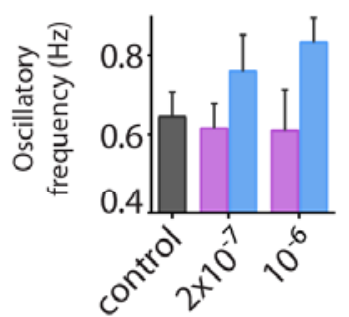

concentration $[\mathrm{M}]$

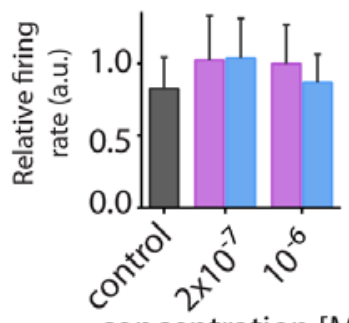

concentration $[\mathrm{M}]$

Figure 4: In vivo photomodulation of brain waves. (A) Representative raw traces of LFP (top) and Multiunit Activity (bottom), showing the differences in OF and relative firing rate between the control, $200 \mathrm{nM}$ and $1 \mu \mathrm{M}$ cis-PAl (pre-irradiated at $365 \mathrm{~nm}$ ) and photoswitching with white light (trans-PAI). (B) Representative spectrogram under control, $200 \mathrm{nM}$ and $1 \mu \mathrm{M}$ cis-PAl (pre-irradiated at $365 \mathrm{~nm}$ ) and photoswitching with white light (trans-PAl). (C) Representative examples of the waveform average of the MUA signal aligned at the Down-to-Up state transition under control, cis-PAl and trans-PAl at different concentrations (see legend). (D) Quantification of $\mathrm{OF}(\mathrm{Hz})$ and relative firing rate $(\mathrm{Hz})$ at different concentrations. 


\section{DISCUSSION}

Different brain states are associated with distinct behaviors. In order to investigate the causality between them, behavioral outcomes must be correlated with the spontaneous and evoked activity in the cortical network. Thus, understanding the mechanisms of brain and behavioral state transitions requires new techniques for the manipulation of neuronal activity. ${ }^{1}$ They must enable the activation and inhibition of specific brain areas and neuronal circuits defined by several complementary criteria, namely electrical stimulation in selected regions, and photomanipulation that is cell-specific, or neurotransmitter-specific (using optogeneticc and photopharmacology, respectively).

Modulation of brain waves is also therapeutically useful in the treatment of CNS diseases, as shown by pioneering techniques used to control cortical activity noninvasively based on electromagnetic stimulation. ${ }^{51,52}$ Transcranial current stimulation enhances motor learning capacity in humans by crossmodulating the oscillatory neural activity (alpha and beta frequencies) in the motor cortex. ${ }^{53-57}$ Transcranial magnetic stimulation can normalize excessive gamma oscillations in the prefrontal cortex, and restore cognitive performance in patients with schizophrenia, Alzheimer's and Parkinson's diseases, ${ }^{58,59}$ and anxiety disorders. ${ }^{60,61}$ However, the physiological mechanisms of brain wave modulation are not known, and they are essential if we are to improve its spatiotemporal and spectral performance, both for fundamental and therapeutic purposes. ${ }^{62}$

Optogenetics ${ }^{63-66}$ has emerged as an alternative to controlling brain waves with electromagnetic fields: photocontrolling the release of $\mathrm{ACh}$, which strongly modulates the transitions between different brain states, $^{9,12}$ is possible by overexpressing photosensitive proteins in cholinergic neurons of mice neocortex. ${ }^{10,67,68}$ However, genetic manipulation is required in this approach. The light-dependent cholinergic muscarinic ligand presented here is, to date, the only way to directly photomodulate cholinergic pathways in intact tissue. We first studied the effect of the superagonist Iperoxo ${ }^{40}$ on isolated cortical slices (Fig. 1) in order to demonstrate that deep-sleep brain states can be controlled by selectively manipulating 
muscarinic receptors at their physiological location and context. The oscillatory frequency of the network was already greatly increased at $100 \mathrm{nM}$ Iperoxo, and led to periods of seizure-like discharges, in agreement with previous studies performed using knockout mice and pilocarpine ${ }^{44,45}$ in which activation of muscarinic acetylcholine receptors induced seizures. Photocontrol of muscarinic signaling was subsequently achieved in vitro and in vivo with the photochromic iperoxo derivative $\mathrm{PAI}^{26}$ which is targeted allosterically at $\mathrm{M} 2$ subtype receptors (Fig. 2-4). In particular, PAI is a dualsteric ligand displaying full agonism, which is probably important to achieve effective photocontrol in slices, where axons from cholinergic afferents have been cut and the concentration of ACh can be assumed to be zero due to the degradation by extracellular esterases. M2 mAChRs are involved in several CNS diseases like major depressive ${ }^{25,69}$ and bipolar disorders, ${ }^{25,70}$ Parkinson's $\mathrm{s}^{25,71,72}$ and Alzheimer's ${ }^{25,72,73}$ diseases, but also in alcohol, smoking and drug dependence. ${ }^{25,74,75}$ These disorders are thus susceptible to drug-based photomodulation in vivo without requiring genetic manipulation. Although PAl cannot cross the blood-brain barrier due to its charge, and its safety profile has not been systematically characterized, it is less likely to trigger immune reactions and mutagenesis than microbial opsins.

In summary, the manipulation of brain state transitions, by means of photocontrolling the frequency of cortical oscillations, has been achieved with a photoswitchable dualsteric agonist of M2 mAChRs. This result opens the way to (1) dissecting the spatiotemporal distribution and pharmacology of brain states, namely how they depend on agonists, antagonists, and modulators of the different muscarinic subtypes expressed in the CNS, and (2) investigating the neuronal dynamics that regulate brain state transitions in the cortical surface and beyond. In particular, two-photon stimulation of PAI using pulsed infrared light ${ }^{26}$ offers the promise of subcellular resolution in three dimensions, ${ }^{76}$ as recently demonstrated with endogenous mGlu ${ }_{5}{ }^{77}$ Compared to the local and often inhomogeneous expression patterns achieved with viral injections of optogenetic constructs, diffusible small molecules like PAI can in principle be applied to larger brain regions to control neuronal oscillations. ${ }^{68}$ Thus, remote control of brain waves based on the photopharmacological manipulation of endogenous muscarinic receptors may reveal the complex 3D molecular signaling 
underlying brain states and their transitions, in order to link them with cognition and behavior in a diversity of wild-type organisms.

\section{EXPERIMENTAL PROCEDURES}

\section{Slice Preparation}

Twenty-five ferrets (4- to 6-month-old) were anesthetized with sodium pentobarbital $(40 \square \mathrm{mg} / \mathrm{kg}$ ) and decapitated. The entire forebrain was rapidly removed and placed in oxygenated cold $\left(4-10 \square{ }^{\circ} \mathrm{C}\right)$ bathing medium. ${ }^{78}$ Ferrets were treated in accordance with protocols approved by the Animal Ethics Committee of the University of Barcelona, which comply with the European Union guidelines on the protection of vertebrates used for experimentation (Directive 2010/63/EU of the European Parliament and the Council of 22 September 2010). Coronal slices (400 $\mu \mathrm{m}$ thick) from primary visual cortex (V1) were used. ${ }^{79}$ To increase tissue viability we used a modification of the sucrose-substitution technique. ${ }^{80}$ During slice preparation, the tissue was placed in a solution in which $\mathrm{NaCl}$ was replaced with sucrose while maintaining the same osmolarity. After preparation, the slices were placed in an interface-style recording chamber (Fine Sciences Tools, Foster City, CA, USA). During the first $30 \square$ min the cortical slices were superfused with an equal mixture in volume of the normal bathing medium, artificial cerebral spinal fluid (ACSF) and the sucrosesubstituted solution. Following this, normal bathing medium was added to the recording chamber and the slices were superfused for $1-2 \square \mathrm{h}$; the normal bathing medium contained (in $\mathrm{mM}$ ): $\mathrm{NaCl}, 126 ; \mathrm{KCl}, 2.5$; $\mathrm{MgSO}_{4}, 2 ; \mathrm{Na}_{2} \mathrm{HPO}_{4}, 1 ; \mathrm{CaCl}_{2}, 2 ; \mathrm{NaHCO}_{3}, 26$; dextrose, 10; and was aerated with $95 \% \mathrm{O}_{2}, 5 \% \mathrm{CO}_{2}$ to a final pH of 7.4. Then, a modified slice solution was used throughout the rest of the experiment; it had the same ionic composition except for different levels of the following (in $\mathrm{mM}$ ): $\mathrm{KCl}, 4 ; \mathrm{MgSO}_{4}, 1$; and $\mathrm{CaCl}_{2}, 1 .^{78} \mathrm{Bath}$ temperature was maintained at $34-36 \square^{\circ} \mathrm{C}$. 


\section{Drug application and photostimulation in brain slices}

Iperoxo and PAI, both prepared as previously reported from commercially available starting materials, ${ }^{26}$ were bath-applied at the concentrations range of $1 \mathrm{nM}$ to $100 \mathrm{nM}$ for Iperoxo and $10 \mathrm{nM}$ to $1 \mu \mathrm{M}$ for PAl, as is mentioned in the Results section. We typically waited more than $1000 \mathrm{~s}$ after the application of the drug in order to let it act, to obtain a stable pattern of electrical activity, and to ensure a stable concentration in the bath. PAI effectively photomodulates the activity of $\mathrm{M} 2$ receptors in vitro and in vivo: in its dark-adapted state (trans form) it behaves as a strong M2 agonist, then upon illumination with UV light (365 nm), PAI switches to its off-state (cis form). PAI can be switched back to its on-state with blue or white light, or using two-photon excitation with pulsed NIR light. ${ }^{26}$ The high thermal stability of PAI inactive form (cis isomer) allows the administration of the inactive drug and subsequent activation of $M 2$ receptors in the target region with white light. ${ }^{26}$ We first investigated the efficacy of PAI in cortical neuronal circuits in vitro by obtaining the dose-response curves of trans- and cis-PAl solutions applied separately. The more active PAI isomer (trans) was tested by applying its dark-adapted form ( $87 \%$ of trans-PAI), and cis-PAl was obtained by illuminating $1 \mathrm{mM}$ stock solutions with $365 \mathrm{~nm}$ light for $10 \mathrm{~min}$ (73\% of cis-PAl). ${ }^{26}$ Increasing concentrations of both trans- and cis-PAI (10 nM, $100 \mathrm{nM}, 300 \mathrm{nM}$ and $1 \mu \mathrm{M})$ were bath applied in order to build up the dose-response curves.

\section{LFP recording and data analysis from in vitro recordings}

LFP recordings started after allowing at least $2 \mathrm{~h}$ of recovery of the slices. Extracellular multiple unit recordings were obtained with flexible arrays of 16 electrodes arranged in columns as in Figure $1 \mathrm{~A}$. The multielectrode array (MEA) covered most of the area occupied by a cortical slice. It consisted of six groups of electrodes positioned to record electrophysiological activity from superficial and from deep cortical layers (692 $\mu \mathrm{m}$ apart) and from what should correspond to three different cortical columns (1500 $\mu \mathrm{m}$ apart). The unfiltered field potential (raw signal) was acquired at $10 \mathrm{kHz}$ with a Multichannel System Amplifier (MCS, Reutlingen, Germany) and digitized with a 1401 CED acquisition board and Spike2 software (Cambridge Electronic Design, Cambridge, UK). The multiunit activity (MUA) was estimated as the power change in the Fourier components at high frequencies from the recorded LFP. ${ }^{81} \mathrm{Up}$-state detection was performed by 
setting a threshold in the $\log (\mathrm{MUA})$ time series as previously described to quantify frequency of the slow oscillations. ${ }^{6,43}$ Relative firing rate (FR) of the Up-states were quantified from the transformed $\log (\mathrm{MUA})$ signal as mean of absolute value of $\log (\mathrm{MUA})$. To study the variability of power spectral densities (PSD) of the local field potential, we used Welch's method with $50 \%$ overlapped Hamming window with a resolution of $1 \mathrm{~Hz}$. All off-line estimates and analyses were implemented in MATLAB (The MathWorks Inc., Natick, MA, USA). All variables in the experimental conditions were compared with the control (no chemical added) condition.

\section{The in vivo preparation}

Cortical electrophysiology experiments were carried out in 2-3-month-old C57BL6/JR mice $(n=4)$ in accordance with the European Union Directive 2010/63/EU and approved by the local ethics committee. Mice were kept under standard conditions (room temperature, 12:12-h light-dark cycle, lights on at 08:00 a.m). Anesthesia was induced by intraperitoneal injection of ketamine $(30 \mathrm{mg} / \mathrm{kg})$ and medetomidine $(100$ $\mathrm{mg} / \mathrm{kg}$ ). After this procedure, the mouse was placed in a stereotaxic frame, and air was enriched with oxygen. Body temperature was maintained at $37^{\circ} \mathrm{C}$ throughout the experiment. ${ }^{6}$ A craniotomy was performed in each mouse: AP $-2.5 \mathrm{~mm}, \mathrm{~L} 1.5 \mathrm{~mm}$ (primary visual cortex, V1). ${ }^{82}$ Cortical recordings were obtained from infragranular layers with 1-2 $M \Omega$ single tungsten electrode insulated with a plastic coating except for the tip (FHC, Bowdoin, ME, USA). Spontaneous local field potential (LFP) recordings from the V1 area provided information about the local neuronal population activity-within $250 \mu \mathrm{m} .{ }^{83}$ MUA estimation, Up-state detection and quantification of relative FR was performed as previously described. All these parameters were used to compare spontaneous activity during anesthesia (control), after application of the pre-illuminated, inactive drug form (cis-PAI) and application of white light to activate the drug (trans-PAI). Cis-PAI was locally delivered to the cerebral cortex surface and activity was recorded while applying a commercial red filter on the white light source to avoid the activation of the drug. The uncovered brain was illuminated with a white light source (Photonic Optics ${ }^{\mathrm{TM}}$ Optics Cold Light Source LED F1) in order to activate the drug in situ (trans-PAl). The electrophysiological signal was amplified with a multichannel system (Multi 
Channel Systems), digitized at $20 \mathrm{kHz}$ with a CED acquisition board and acquired with Spike 2 software (Cambridge Electronic Design) unfiltered. ${ }^{84}$

\section{Statistical analysis for slice experiments}

Both in vitro and in vivo oscillatory frequency (OF) are reported as mean \pm SEM. Measurements under different conditions were compared using the Friedman test and the Wilcoxon post-hoc tests corrected for multiple comparisons. $^{85}$

\section{Acknowledgements}

We thank Miquel Bosch for comments on the manuscript. This research received funding from European Union Research and Innovation Programme Horizon 2020 (Human Brain Project SGA2 Grant Agreement 785907, WaveScalES), European Research ERA-Net SynBio programme (Modulightor project), and financial support from Agency for Management of University and Research Grants/Generalitat de Catalunya (CERCA Programme; 2017-SGR-1442 project; RIS3CAT plan), Fonds Européen de Développement Économique et Régional (FEDER) funds, Ministry of Economy and Competitiveness (MINECO)/FEDER (Grant CTQ201680066-R to PG and BFU2017-85048-R to MVSV), and the Fundaluce foundation.

\section{References}

1. Andalman, A. S. et al. Neuronal Dynamics Regulating Brain and Behavioral State Transitions. Cell 177, 970-985.e20 (2019).

2. Wang, S. et al. Neurons in the human amygdala selective for perceived emotion. Proc. Natl. Acad. Sci. U. S. A. 111, E3110-9 (2014).

3. Krehbiel, D., Bartel, B., Dirks, M. \& Wiens, W. Behavior and brain neurotransmitters: Correlations in different strains of mice. Behav. Neural Biol. 46, 30-45 (1986). 
4. Hanin, I. Central neurotransmitter function and its behavioral correlates in man. Environ. Health Perspect. 26, 135-141 (1978).

5. Peyrache, A. et al. Spatiotemporal dynamics of neocortical excitation and inhibition during human sleep. Proc. Natl. Acad. Sci. U. S. A. 109, 1731-6 (2012).

6. Ruiz-Mejias, M., Ciria-Suarez, L., Mattia, M. \& Sanchez-Vives, M. V. Slow and fast rhythms generated in the cerebral cortex of the anesthetized mouse. J. Neurophysiol. 106, 2910-21 (2011).

7. Sanchez-Vives, M. V \& Mattia, M. Slow wave activity as the default mode of the cerebral cortex. Arch. Ital. Biol. 152, 147-55.

8. Muller, L., Reynaud, A., Chavane, F. \& Destexhe, A. The stimulus-evoked population response in visual cortex of awake monkey is a propagating wave. Nat. Commun. 5, 3675 (2014).

9. Lee, S.-H. \& Dan, Y. Neuromodulation of Brain States. Neuron 76, 209-222 (2012).

10. Pinto, L. et al. Fast modulation of visual perception by basal forebrain cholinergic neurons. Nat. Neurosci. 16, 1857-1863 (2013).

11. Ramaswamy, S., Colangelo, C. \& Markram, H. Data-Driven Modeling of Cholinergic Modulation of Neural Microcircuits: Bridging Neurons, Synapses and Network Activity. Front. Neural Circuits 12, 77 (2018).

12. Hasselmo, M. E. \& Sarter, M. Modes and Models of Forebrain Cholinergic Neuromodulation of Cognition. Neuropsychopharmacology 36, 52-73 (2011).

13. Buzsàki, G. \& Gage, F. H. The cholinergic nucleus basalis: a key structure in neocortical arousal. EXS 57, 159-71 (1989).

14. Herrero, J. L. et al. Acetylcholine contributes through muscarinic receptors to attentional modulation in V1. Nature 454, 1110-1114 (2008).

15. Conner, J. M., Culberson, A., Packowski, C., Chiba, A. A. \& Tuszynski, M. H. Lesions of the Basal 
forebrain cholinergic system impair task acquisition and abolish cortical plasticity associated with motor skill learning. Neuron 38, 819-29 (2003).

16. Kilgard, M. Cholinergic modulation of skill learning and plasticity. Neuron 38, 678-80 (2003).

17. Ramanathan, D., Tuszynski, M. H. \& Conner, J. M. The Basal Forebrain Cholinergic System Is Required Specifically for Behaviorally Mediated Cortical Map Plasticity. J. Neurosci. 29, 5992-6000 (2009).

18. Winkler, J., Suhr, S. T., Gage, F. H., Thal, L. J. \& Fisher, L. J. Essential role of neocortical acetylcholine in spatial memory. Nature 375, 484-487 (1995).

19. Berg, R. W., Friedman, B., Schroeder, L. F. \& Kleinfeld, D. Activation of Nucleus Basalis Facilitates Cortical Control of a Brain Stem Motor Program. J. Neurophysiol. 94, 699-711 (2005).

20. Kilgard, M. P. \& Merzenich, M. M. Cortical Map Reorganization Enabled by Nucleus Basalis Activity. Science (80-. ). 279, 1714-1718 (1998).

21. Henny, P. \& Jones, B. E. Projections from basal forebrain to prefrontal cortex comprise cholinergic, GABAergic and glutamatergic inputs to pyramidal cells or interneurons. Eur. J. Neurosci. 27, 654-670 (2008).

22. Donthamsetti, P. C. et al. Genetically Targeted Optical Control of an Endogenous G Protein-Coupled Receptor. J. Am. Chem. Soc. 141, 11522-11530 (2019).

23. Yamaguchi, H. \& de Lecea, L. In vivo cell type-specific CRISPR gene editing for sleep research. Journal of Neuroscience Methods vol. 316 99-102 (2019).

24. Miesenböck, G. Optogenetic Control of Cells and Circuits. Annu. Rev. Cell Dev. Biol. 27, 731-758 (2011).

25. Scarr, E. Muscarinic Receptors: Their Roles in Disorders of the Central Nervous System and Potential as Therapeutic Targets. CNS Neurosci. Ther. 18, 369-379 (2012).

26. Riefolo, F. et al. Optical Control of Cardiac Function with a Photoswitchable Muscarinic Agonist. J. Am. 
Chem. Soc. 141, 7628-7636 (2019).

27. McCormick, D. A. Cellular mechanisms underlying cholinergic and noradrenergic modulation of neuronal firing mode in the cat and guinea pig dorsal lateral geniculate nucleus. J. Neurosci. 12, 27889 (1992).

28. Steriade, M., Amzica, F. \& Nunez, A. Cholinergic and noradrenergic modulation of the slow (approximately $0.3 \mathrm{~Hz}$ ) oscillation in neocortical cells. J. Neurophysiol. 70, 1385-1400 (1993).

29. Steriade, M. Cholinergic blockage of network- and intrinsically generated slow oscillations promotes waking and REM sleep activity patterns in thalamic and cortical neurons. Prog. Brain Res. 98, 345-55 (1993).

30. Jacobs, S. E. \& Juliano, S. L. The impact of basal forebrain lesions on the ability of rats to perform a sensory discrimination task involving barrel cortex. J. Neurosci. 15, 1099-109 (1995).

31. Xiang, Z., Huguenard, J. R. \& Prince, D. A. Cholinergic switching within neocortical inhibitory networks. Science 281, 985-8 (1998).

32. Picciotto, M. R., Higley, M. J. \& Mineur, Y. S. Acetylcholine as a neuromodulator: cholinergic signaling shapes nervous system function and behavior. Neuron 76, 116-29 (2012).

33. Zagha, E. \& McCormick, D. A. Neural control of brain state. Curr. Opin. Neurobiol. 29, 178-86 (2014).

34. Kuczewski, N., Aztiria, E., Gautam, D., Wess, J. \& Domenici, L. Acetylcholine modulates cortical synaptic transmission via different muscarinic receptors, as studied with receptor knockout mice. J. Physiol. 566, 907-919 (2005).

35. Wester, J. C. \& Contreras, D. Differential modulation of spontaneous and evoked thalamocortical network activity by acetylcholine level in vitro. J. Neurosci. 33, 17951-66 (2013).

36. Compte, A. et al. Spontaneous High-Frequency $(10-80 \mathrm{~Hz})$ Oscillations during Up States in the Cerebral Cortex In Vitro. J. Neurosci. 28, 13828-13844 (2008). 
37. Schmidt, S. L., Chew, E. Y., Bennett, D. V., Hammad, M. A. \& Fröhlich, F. Differential effects of cholinergic and noradrenergic neuromodulation on spontaneous cortical network dynamics. Neuropharmacology 72, 259-273 (2013).

38. Castro-Alamancos, M. A. \& Gulati, T. Neuromodulators produce distinct activated states in neocortex. J. Neurosci. 34, 12353-67 (2014).

39. McCormick, D. A. \& Williamson, A. Convergence and divergence of neurotransmitter action in human cerebral cortex. Proc. Natl. Acad. Sci. U. S. A. 86, 8098-102 (1989).

40. Barocelli, E. et al. New analogues of oxotremorine and oxotremorine-M: estimation of their in vitro affinity and efficacy at muscarinic receptor subtypes. Life Sci. 67, 717-23 (2000).

41. Schrage, R. et al. Agonists with supraphysiological efficacy at the muscarinic M2 ACh receptor. Br. J. Pharmacol. 169, 357-70 (2013).

42. Sanchez-Vives, M. V. \& McCormick, D. A. Cellular and network mechanisms of rhytmic recurrent activity in neocortex. Nat. Neurosci. 3, 1027-1034 (2000).

43. Sanchez-Vives, M. V et al. Inhibitory modulation of cortical up states. J. Neurophysiol. 104, 1314-24 (2010).

44. Yi, F. et al. Muscarinic excitation of parvalbumin-positive interneurons contributes to the severity of pilocarpine-induced seizures. Epilepsia 56, 297-309 (2015).

45. Hamilton, S. E. et al. Disruption of the $\mathrm{m} 1$ receptor gene ablates muscarinic receptor-dependent $\mathrm{M}$ current regulation and seizure activity in mice. Proc. Natl. Acad. Sci. 94, 13311-13316 (1997).

46. Steriade, M., Contreras, D., Curró Dossi, R. \& Nuñez, A. The slow $(<1 \mathrm{~Hz})$ oscillation in reticular thalamic and thalamocortical neurons: scenario of sleep rhythm generation in interacting thalamic and neocortical networks. J. Neurosci. 13, 3284-99 (1993).

47. Sakata, S. \& Harris, K. D. Laminar Structure of Spontaneous and Sensory-Evoked Population Activity in 
Auditory Cortex. Neuron 64, 404-418 (2009).

48. Chauvette, S., Volgushev, M. \& Timofeev, I. Origin of active states in local neocortical networks during slow sleep oscillation. Cereb. Cortex 20, 2660-2674 (2010).

49. Capone, C. et al. Slow waves in cortical slices: How spontaneous activity is shaped by laminar structure. Cereb. Cortex 29, 319-335 (2019).

50. Beltramo, R. et al. Layer-specific excitatory circuits differentially control recurrent network dynamics in the neocortex. Nat. Neurosci. 16, 227-234 (2013).

51. Antal, A. et al. Comparatively weak after-effects of transcranial alternating current stimulation (tACS) on cortical excitability in humans. Brain Stimul. 1, 97-105 (2008).

52. Terney, D., Chaieb, L., Moliadze, V., Antal, A. \& Paulus, W. Increasing Human Brain Excitability by Transcranial High-Frequency Random Noise Stimulation. J. Neurosci. 28, 14147-14155 (2008).

53. Sugata, H. et al. Modulation of Motor Learning Capacity by Transcranial Alternating Current Stimulation. Neuroscience 391, 131-139 (2018).

54. Pollok, B., Boysen, A.-C. \& Krause, V. The effect of transcranial alternating current stimulation (tACS) at alpha and beta frequency on motor learning. Behav. Brain Res. 293, 234-240 (2015).

55. Joundi, R. A., Jenkinson, N., Brittain, J.-S., Aziz, T. Z. \& Brown, P. Driving Oscillatory Activity in the Human Cortex Enhances Motor Performance. Curr. Biol. 22, 403-407 (2012).

56. Pogosyan, A., Gaynor, L. D., Eusebio, A. \& Brown, P. Boosting Cortical Activity at Beta-Band Frequencies Slows Movement in Humans. Curr. Biol. 19, 1637-1641 (2009).

57. Moisa, M., Polania, R., Grueschow, M. \& Ruff, C. C. Brain Network Mechanisms Underlying Motor Enhancement by Transcranial Entrainment of Gamma Oscillations. J. Neurosci. 36, 12053-12065 (2016).

58. Pereira, L. S., Müller, V. T., da Mota Gomes, M., Rotenberg, A. \& Fregni, F. Safety of repetitive 
transcranial magnetic stimulation in patients with epilepsy: A systematic review. Epilepsy Behav. 57, 167-176 (2016).

59. Lefaucheur, J.-P. Treatment of Parkinson's disease by cortical stimulation. Expert Rev. Neurother. 9, 1755-71 (2009).

60. Xiao, Z., Li, C. \& Wang, J. Repetitive transcranial magnetic stimulation (rTMS) for panic disorder. in Cochrane Database of Systematic Reviews (ed. Wang, J.) (John Wiley \& Sons, Ltd, 2011). doi:10.1002/14651858.CD009083.

61. Lefaucheur, J.-P. et al. Evidence-based guidelines on the therapeutic use of repetitive transcranial magnetic stimulation (rTMS). Clin. Neurophysiol. 125, 2150-2206 (2014).

62. Paulus, W. Transcranial brain stimulation: potential and limitations. e-Neuroforum 5, 29-36 (2014).

63. Cardin, J. A. et al. Driving fast-spiking cells induces gamma rhythm and controls sensory responses. Nature 459, 663-667 (2009).

64. Sohal, V. S., Zhang, F., Yizhar, O. \& Deisseroth, K. Parvalbumin neurons and gamma rhythms enhance cortical circuit performance. Nature 459, 698-702 (2009).

65. Adesnik, H. \& Naka, A. Cracking the Function of Layers in the Sensory Cortex. Neuron vol. $1001028-$ $1043(2018)$.

66. Naka, A. et al. Complementary networks of cortical somatostatin interneurons enforce layer specific control. Elife 8, (2019).

67. Zucca, S., Pasquale, V., Lagomarsino de Leon Roig, P., Panzeri, S. \& Fellin, T. Thalamic Drive of Cortical Parvalbumin-Positive Interneurons during Down States in Anesthetized Mice. Curr. Biol. 29, 14811490.e6 (2019).

68. Zucca, S. et al. An inhibitory gate for state transition in cortex. Elife 6, (2017).

69. Gibbons, A. S., Scarr, E., McLean, C., Sundram, S. \& Dean, B. Decreased muscarinic receptor binding in 
the frontal cortex of bipolar disorder and major depressive disorder subjects. J. Affect. Disord. 116, 184-91 (2009).

70. Cannon, D. M. et al. Reduced Muscarinic Type 2 Receptor Binding in Subjects With Bipolar Disorder. Arch. Gen. Psychiatry 63, 741 (2006).

71. Warren, N. M., Piggott, M. A., Lees, A. J. \& Burn, D. J. The basal ganglia cholinergic neurochemistry of progressive supranuclear palsy and other neurodegenerative diseases. J. Neurol. Neurosurg.

Psychiatry 78, 571 (2007).

72. Piggott, M. A. et al. Muscarinic receptors in basal ganglia in dementia with Lewy bodies, Parkinson's disease and Alzheimer's disease. J. Chem. Neuroanat. 25, 161-73 (2003).

73. Quirion, R. Cholinergic markers in Alzheimer disease and the autoregulation of acetylcholine release. J. Psychiatry Neurosci. 18, 226-34 (1993).

74. Dick, D. M. et al. Alcohol dependence with comorbid drug dependence: genetic and phenotypic associations suggest a more severe form of the disorder with stronger genetic contribution to risk. Addiction 102, 1131-1139 (2007).

75. Mobascher, A. et al. Association of a variant in the muscarinic acetylcholine receptor 2 gene (CHRM2) with nicotine addiction. Am. J. Med. Genet. Part B Neuropsychiatr. Genet. 153B, 684-690 (2010).

76. Wenzel, M., Hamm, J. P., Peterka, D. S. \& Yuste, R. Acute Focal Seizures Start As Local Synchronizations of Neuronal Ensembles. J. Neurosci. 39, 8562-8575 (2019).

77. Pittolo, S. et al. Reversible silencing of endogenous receptors in intact brain tissue using 2-photon pharmacology. Proc. Natl. Acad. Sci. 116, 13680-13689 (2019).

78. Mattia, M. \& Sanchez-Vives, M. V. Exploring the spectrum of dynamical regimes and timescales in spontaneous cortical activity. Cogn. Neurodyn. 6, 239-250 (2012).

79. Krimer, L. S. \& Goldman-Rakic, P. S. Prefrontal microcircuits: membrane properties and excitatory 
input of local, medium, and wide arbor interneurons. J. Neurosci. 21, 3788-96 (2001).

80. Aghajanian, G. K. \& Rasmussen, K. Intracellular studies in the facial nucleus illustrating a simple new method for obtaining viable motoneurons in adult rat brain slices. Synapse 3, 331-338 (1989).

81. Mattia, M. \& Del Giudice, P. Population dynamics of interacting spiking neurons. Phys. Rev. E 66, 051917 (2002)

82. Golmohammadi, M. G. et al. Comparative Analysis of the Frequency and Distribution of Stem and Progenitor Cells in the Adult Mouse Brain. Stem Cells 26, 979-987 (2008).

83. Katzner, S. et al. Local origin of field potentials in visual cortex. Neuron 61, 35-41 (2009).

84. Castano-Prat, P., Perez-Zabalza, M., Perez-Mendez, L., Escorihuela, R. M. \& Sanchez-Vives, M. V. Slow and Fast Neocortical Oscillations in the Senescence-Accelerated Mouse Model SAMP8. Front. Aging Neurosci. 9, 141 (2017).

85. Benjamini, Y. \& Hochberg, Y. Controlling the False Discovery Rate: A Practical and Powerful Approach to Multiple Testing. Journal of the Royal Statistical Society. Series B (Methodological) vol. 57 289-300 (1995). 